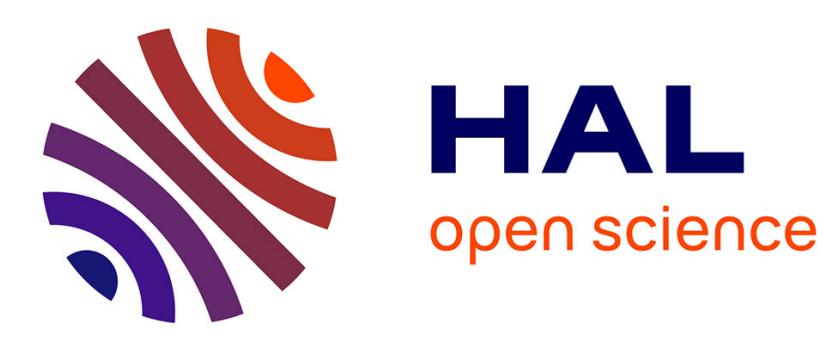

\title{
About string stability of a vehicle chain with unidirectional controller
}

\author{
Arash Farnam, Alain Sarlette
}

\section{To cite this version:}

Arash Farnam, Alain Sarlette. About string stability of a vehicle chain with unidirectional controller.

European Journal of Control, 2019, 10.1016/j.ejcon.2019.03.002 . hal-02394705

\section{HAL Id: hal-02394705 \\ https://hal.inria.fr/hal-02394705}

Submitted on 4 Dec 2019

HAL is a multi-disciplinary open access archive for the deposit and dissemination of scientific research documents, whether they are published or not. The documents may come from teaching and research institutions in France or abroad, or from public or private research centers.
L'archive ouverte pluridisciplinaire HAL, est destinée au dépôt et à la diffusion de documents scientifiques de niveau recherche, publiés ou non, émanant des établissements d'enseignement et de recherche français ou étrangers, des laboratoires publics ou privés. 


\title{
About string stability of a vehicle chain with unidirectional controller
}

\author{
Arash Farnam* and Alain Sarlette ${ }^{\dagger}$
}

February 18, 2019

\begin{abstract}
This paper deals with the problem of string stability in a chain of accelerationcontrolled vehicles. It is known that string stability cannot be achieved, with any linear controller, when the vehicles' control inputs are based only on relative distances to a fixed number of predecessors. We extend the set of characteristics under which string stability is impossible by including elements like dynamic sensor parts and local intervehicular communication, as in cooperative adaptive cruise control. It is also known that a weaker form of string stability is achievable by adding absolute velocity measurements (e.g. "time-headway" policy). We show that a stronger property can also be achieved, provided steady-state control gain is infinite e.g. by using integral control.
\end{abstract}

\section{Introduction}

Grouping vehicles into tight platoons is a method for increasing the capacity of roads by automated highway systems (Chu (1974)). The distances between vehicles is decreased by ensuring safety thanks to automatic controllers, enabling many vehicles to accelerate or brake simultaneously and eliminating the distance needed for human reaction. The most fundamental platoon is the vehicle chain, where all vehicles are aligned after each other. During the recent years numerous works have considered different control strategies to stabilize each vehicle at a desired distance from its predecessor and follower (Chu (1974); Stüdli et al. (2017); Sheikholeslam and Desoer (1990); Levine and Athans (1996); Rogge and Aeyels (2008)).

When disturbance inputs affect the vehicle chain, its a priori cooperative coupling can lead to new types of instabilities. In particular, string instability is a situation where the spacing error between consecutive vehicles grows unbounded as the number of vehicles increases to infinity. Since its definition in Swaroop and Hedrick (1996); Swaroop (1994), string (in)stability has spurred a lot of discussion and research. Indeed, it is well known since Swaroop (1994) that string stability cannot be achieved in a homogeneous string of interconnected secondorder integrators (e.g. acceleration-controlled vehicles), with any controller that is linear and whose local control actions are determined from the relative distance to a few directly preceding vehicles: some perturbations will unavoidably grow unbounded along the chain. This

${ }^{*}$ ID Lab, Department of Electronics and Information Systems (ELIS), Faculty of Engineering and Architecture, Ghent University; Technologiepark Zwijnaarde 914, 9052 Zwijnaarde(Ghent), Belgium (e-mail: arash.farnam@ugent.be).

${ }^{\dagger}$ ELIS, Ghent University, Belgium; and QUANTIC lab, INRIA Paris, France (e-mail: alain.sarlette@inria.fr) 
has attracted attention as a prototypical, unavoidable shortcoming of linear systems (Sheikholeslam and Desoer (1990); Levine and Athans (1996)). When the controller reacts to the just preceding vehicle only, the unavoidability of string instability follows essentially from the Bode integral for the transfer function from vehicle $i-1$ to $i$, which takes the form of a complementary sensitivity function. This has led to first observations about how a disturbance gets amplified from one vehicle to the next, without bound as the chain gets longer. However, it has soon been noted that the problem is more general and that also with other types of couplings where the dynamics cannot be reduced to propagation of disturbances from $i-1$ to $i$ - e.g. with bidirectional coupling Barooah and Hespanha (2005); Seiler et al. (2004); Herman et al. (2017) - small disturbances on initial conditions or in the inputs can lead to big errors on inter-vehicle spacing, more precisely unbounded errors as the number of vehicles tends to infinity. This has led to the more general concept of string instability. The latter has also become a relevant concept beyond the strict application to road vehicles, for the distributed control of chains of abstract subsystems, with particular types of open-loop dynamics and control inputs depending on local relative output measurements. We will use the road vehicle chain as a guiding example, but keeping in mind that the relevance of possible settings or assumptions should not be restricted to this particular application.

To solve the string instability issue, a notable alternative setting is to allow the use of absolute velocity in the controller, see Rogge and Aeyels (2008), Klinge and Middleton (2009), Knorn et al. (2014). It has been shown that the effect of a disturbance from the leading vehicle can then be kept in check for arbitrarily long chains. Another natural feature is to allow local communication, as in Cooperative Adaptive Cruise Control (CACC, see Ploeg et al. (2014), Öncü et al. (2014), Ploeg et al. (2014), Ploeg et al. (2015), Milanés and Shladover (2014)). Remarkably, the literature considering communication also always assumes the use of absolute velocity in the controller, and it thus remains unclear what can be done with communication but without using absolute velocity.

The aim of the present paper is to add essentially two types of precision to this picture. First, we consider countering the effect of disturbances acting possibly on all vehicles in the $\left(L_{2}, l_{2}\right)$ sense. This is in agreement with the more general concept of string stability, and with the practical concern of avoiding catastrophic effects in long chains due to small errors in all the vehicles' inputs. For this, we give a positive result using PID control and absolute velocity. Second, we clarify how alternative settings behave in absence of absolute velocity or integral action in the controller. For this, we provide a series of negative results; in particular the use of absolute velocity appears necessary even in presence of CACC-type communication. A more detailed statement of state of the art and of our contributions is given in Section 3, after clarifying the setting.

While the present work focuses on unidirectional vehicle chains - i.e. vehicles react to their predecessors only - a whole line of work has been developed for bidirectional chains as well, where vehicles react to predecessors and followers. Impossibilities to satisfy string stability have been obtained for both symmetric (Barooah and Hespanha (2005); Seiler et al. (2004)) and asymmetric (Herman et al. (2017)) couplings. Conversely, Hao and Barooah (2013); Yamamoto and Smith (2015) have identified the possibility to avoid unbounded growth of a disturbance that acts on the leader only, without resorting to absolute velocity; this result thus fundamentally differs from the impossibility implied by the Bode integral for unidirectional chains. Our viewpoint on the bidirectional setting can be found in Farnam (2018).

The paper is organized as follows. Section 2 describes the string stability property to be investigated and the various control settings which we analyze. Section 3 gives the summary 
of our results. The proofs are given in Appendix.

\section{Problem Setting}

\subsection{Open-loop model and control objective}

Consider a chain of $N+1$ subsystems (e.g. vehicles) with respective configuration (e.g. deviation from nominal position) $x_{i} \in \mathbb{R}$, for $i=0,1,2, \ldots, N$. They move according to the second-order integrator dynamics, expressed in Laplace domain:

$$
s^{2} x_{i}(s)=u_{i}(s)+d_{i}(s)
$$

where $u_{i}$ is the control input, and $d_{i}$ is a disturbance input signal. These disturbance signals can also be an indirect way to model nonzero initial conditions, which are the focus in other papers. The pure second-order integrator is a standard idealization, valid in good approximation for space vehicles, vacuum transit, after other dynamics has been compensated by local feedback, or when it can be included (e.g. by linearization) in the form of $u_{i}(s)$ that we further specify below.

The goal of stability, on a system like (1) with fixed $N$, is to ensure that arbitrary input signals $d_{i}$ are not amplified unboundedly in the state or output. String stability further checks what happens when $N$ becomes infinite; thus if the system is stable for each $N$ but with stability bounds depending on $N$ in a bad way, then string stability may fail. The standard definition of string stability (Swaroop and Hedrick (1996), Seiler et al. (2004)) considers how the relative distances between consecutive vehicles are affected. In the "timeheadway" extension Klinge and Middleton (2009), the target distance between vehicles is made proportional to their absolute velocity, such that the configuration error ("output signal" of interest) is

$$
e_{i}(s)=x_{i-1}(s)-x_{i}(s)-h s x_{i}(s),
$$

for some $h \geq 0$. Different variants of string stability, see e.g. Stüdli et al. (2017), consider how this error signal is affected either by initial conditions or by disturbance inputs, and measure the signals with different norms. We here focus on the effect of disturbance inputs, and consider two types of 2-norms. For a vector $v$ of signals $v_{i}(t), i=1,2, \ldots, N$, the $L_{2}$ norm of $v_{i}$ is defined as $\left\|v_{i}(.)\right\|_{2}=\sqrt{\int_{-\infty}^{+\infty}\left(v_{i}(t)\right)^{2} d t}$ and the $\left(L_{2}, l_{2}\right)$ norm of $v$ is defined as $\|v(.)\|_{2}=\sqrt{\sum_{i=1}^{N} \int_{-\infty}^{+\infty}\left(v_{i}(t)\right)^{2} d t}$, which by Parseval both have equivalent expressions in frequency domain. When evaluating the vector norm at a specific value of $t$ in time domain or of $s$ in frequency domain we write e.g. $\|v(s)\|_{2}=\sqrt{\sum_{i=1}^{N}\left|v_{i}(s)\right|^{2}}$.

Definition $1\left[\left(L_{2}, l_{2}\right)\right.$ string stability]: The chain (1), controlled with feedback signals $u_{i}$ to be designed, is called $\left(L_{2}, l_{2}\right)$ string stable if there exists a constant value $c_{1}$ such that

$$
\|e(.)\|_{2} \leq c_{1}\|d(.)\|_{2}
$$

for all bounded signals $d$, and all chain lengths $N$.

Definition 2 [ $L_{2}$ string stability]: The chain (1), controlled with feedback signals $u_{i}$ to be designed, is called $L_{2}$ string stable if there exists a constant value $c_{1}$ such that

$$
\left\|e_{k}(.)\right\|_{2} \leq c\left\|d_{i}(.)\right\|_{2} \text { for all } k,
$$


for any situation with bounded disturbance signal $d_{i}$ at some $i$ and zero disturbances on all $k \neq i$, and for all $N$.

The difficulty, for both definitions, is to make the bound uniform in $N$. Definition 2, considered in e.g. Barooah et al. (2009); Hao and Barooah (2013); Yamamoto and Smith (2015); Klinge and Middleton (2009); Knorn et al. (2014); Herman et al. (2017), essentially requires that the transfer function from any $d_{i}$ to any $e_{k}$ is $H$-infinity bounded independently of $N$. This appears to be a desirable necessary property for practical applications, but not strictly sufficient. Indeed, $L_{2}$ string stability does not bound independently of $N$ what happens when disturbances are present at the inputs of all the vehicles: if each individual disturbance $d_{i}$ can induce a nonzero bounded error on each $e_{k}$, without further constraints, then when disturbances act on all the vehicles the sum of their effects could make $e_{k}$ grow unbounded with $N$. Avoiding this is the motivation behind the stronger Definition 1, which is in line with the definitions considered by Stüdli et al. (2017); Barooah and Hespanha (2005); Seiler et al. (2004) among others. Indeed, Definition 1 requires that a bounded disturbance input vector (times $N$ ) should lead to a bounded output error vector (times $N$ ). The true practical concern should probably involve infinity norms both over time and over vehicle indices, in a BIBO sense. The corresponding 2-norms are used as a standard proxy to take advantage of their basis-independence, like in many other control-theoretic performance studies.

\subsection{Constraints on controller design}

We will consider different constraints on the feedback controller design, all revolving around the unavailability of absolute or long-range position measurements. The most general controller that we consider, and motivated in the next paragraph, writes:

$$
\begin{aligned}
u_{i}(s) & =K(s) e_{i}^{\prime}(s)+H(s) r_{i}(s) \\
r_{i}(s) & =W(s) q_{i-1}(s) \\
q_{i}(s) & =F(s) e_{i}^{\prime}(s)+G(s) r_{i}(s) \\
e_{i}^{\prime} & =M^{(r)}(s) x_{i-1}(s)-M^{(f)}(s) x_{i}(s)-h s x_{i}(s),
\end{aligned}
$$

for $i=1,2, \ldots, N$. Here $K(s)$ represents the linear controller's transfer function depending on measured configuration error $e_{i}^{\prime}$, and satisfying $K(0) \neq 0$ (impossibility to have a perfect derivative of the measurement output in order to cancel the pole at $s=0$ in the open-loop system (1)). Furthermore, unlike in Li and De Schutter (2012), we do not allow $K(s)$ to grow unboundedly with $N$ and we assume all transfer functions independent of $N$. For $h=0$, the $e_{i}^{\prime}$ are the measured inter-vehicle distances and they may differ from the true inter-vehicle distance $x_{i-1}(s)-x_{i}(s)$. Examining a simple physical sensor model, without a global reference, even shows that the typical situation does not necessarily correspond to $e_{i}^{\prime}(s)=M(s) e_{i}(s)$ for $h=0$, i.e. a relative distance measurement is not just proportional to the true relative distance. Indeed, consider a distance sensor that measures the time-of-flight between a sensor part mounted at the front of vehicle $i$ and its companion part mounted on the rear of vehicle $i-1$. The mounts of these sensor parts can have some dynamics, that are themselves sensitive to the relative position of a sensor part $p_{i}^{(r)}$ or $p_{i}^{(f)}$ with respect to the vehicle on which it is mounted $x_{i}$. Thus, we should have $s^{2} p_{i}=K^{(\cdot)}(s)\left(x_{i}-p_{i}\right)$, which yields:

$$
M^{(r)}=\frac{K^{(r)}}{s^{2}+K^{(r)}}, \quad M^{(f)}=\frac{K^{(f)}}{s^{2}+K^{(f)}},
$$


with $K^{(r)}(0) \neq 0$ and $K^{(f)}(0) \neq 0$, respectively for the sensor parts mounted on the rear and front ends of the vehicles. In particular, at the limit of infinitely stiff mounts $K^{(r)}, K^{(f)} \rightarrow \infty$, we get

$$
M^{(r)}(s)=M^{(f)}(s)=1
$$

and the controller $u_{i}(s)$ just depends on the configuration error $e_{i}(s)$; if furthermore $h=0$ it reduces to true relative position $e_{i}(s)=x_{i-1}(s)-x_{i}(s)$. In addition, $u_{i}$ relies on a communication signal $r_{i}(s)$ received from the preceding vehicle. Imperfections in the communication channel are taken into account by a simplified linear model, with the bounded transfer function $|W(j \omega)|$ not exactly known. The signal $q_{i}(s)$ sent into this channel by vehicle $i$ is computed as a linear function, on the same basis as the control signal, with $H(s), F(s)$ and $G(s)$ controller transfer functions to be designed. We make the following assumptions on the communication channel.

\section{Assumption 1 [communication channel]:}

- The poles of $F, G, H, W$ must all have negative real parts.

- $G(j \omega), F(j \omega)$ and $\frac{H(j \omega)}{K(j \omega)}$ are bounded for all $\omega$.

- System behavior must be robust to replacing $W$ by any $\tilde{W}$ close to $W$, without adapting the controller $F, G, H, K$.

Let us briefly motivate the various features of (3) and the associated Assumption 1.

- The most basic setting, with $h=0, M^{(r)}(s)=M^{(f)}(s)=1$ and $W=0$ (thus no communication $H=F=G=0$ ), was the initial focus of Swaroop and Hedrick (1996) and is known to be string unstable with any linear controller. To check how alternative settings could help, we stay in the linear realm.

- Just introducing the possibility of $h>0$, was shown to enable satisfying Definition 2 (Klinge and Middleton (2009)). However, the time-headway policy makes the effective inter-vehicle distance velocity-dependent, where absolute velocity of the chain would be defined via some other controller, to be carefully interfaced, and where high velocity would automatically imply large(r) inter-vehicle distance, unlike what a high-capacity vehicle "train" should do. For some vehicle types - think of a platoon of space vehicles — or for abstract subsystems, it also remains to be seen exactly how (accurately) the absolute velocity of each individual would be measured in practice towards implementing (3) with $h>0$. Therefore, it is relevant to check whether other features could allow us to achieve string stability without using absolute velocity in the controller, i.e. with $h=0$.

- The use of dynamic sensor mounts $M^{(r)}(s), M^{(f)}(s)$ is another way of breaking the symmetry of all input commands depending just on $e_{i}=x_{i-1}-x_{i}$. Tuning these dynamics in some beneficial way appears as a less invasive solution, than assuming with $h>0$ that a global reference is available for absolute velocity measurement. We thus see this as another potential opportunity to break the impossibility observed in Swaroop and Hedrick (1996); Swaroop (1994).

- The use of local communication is a natural feature, considered e.g. in Ploeg et al. (2014); Öncü et al. (2014); Ploeg et al. (2014, 2015); Milanés and Shladover (2014), with $h>0$. We want to see how it fares with $h=0$. To give a meaning to "local communication" in continuous-time, it is necessary to specify a communication model of finite capacity. If instead communication was assumed perfect and instantaneous, then communicating $r_{1}=x_{0}-x_{1}$, $r_{i}=r_{i-1}+e_{i}$ for $i>1$, vehicle $i$ would in fact receive $r_{i}=x_{0}-x_{i}$. In other words, vehicle $i$ 
has access to the perfect value of $x_{0}-x_{i}$, as if the coupling with the leader was global. On this basis each vehicle can just stabilize its position with respect to the leader with some stable controller $u_{i}(s)=H(s) r_{i}(s)=H(s)\left(x_{0}-x_{i}\right)$, independently of all the other vehicles in the chain. However, assuming perfect transmission of arbitrary signals throughout the whole vehicle chain is not what a realistic communication channel can do. Assumption 1 formulates a few conditions to make the behavior robust to imperfect communication: the communication system must be internally stable (first item); the encoding and decoding filters must avoid unbounded amplification of possible communication noise, both towards generating the next communicated signal and before applying the controller $K$ (second item); and every controller should work under the premise that we know the communication channel only imperfectly (third item). Another typical limitation would be a finite bandwidth in $W$ associated with additive communication noise, but we do not even need this assumption for our results.

\section{Summary of results}

We now summarize more accurately the known results from the literature and new results of the present paper, see Table 1. Our proofs are given in appendix.

Regarding known results, the first observations of string instability (Swaroop (1994)) were made when each control input $u_{i}$ is reacting just to the relative distance $x_{i-1}-x_{i}$ with the vehicle in front, thus corresponding to (1),(2),(3) with $M^{(r)}=M^{(f)}=1, h=W=0$. It was observed that $L_{2}$ string stability is impossible (Definition 2), and a fortiori $\left(L_{2}, l_{2}\right)$ string stability (Definition 1) as well, with any linear controller that avoids pole cancellation $(K(0) \neq$ $0)$. Let us repeat the short proof of this first observation, which has guided researchers in their search for alternative control strategies. Let $u_{i}(s)=K(s) e_{i}(s)$. From (1), the closed-loop equation for the $e_{i}$ and with a disturbance on the first subsystem only writes

$$
e_{i}=T(s)^{i-1} \frac{1}{s^{2}+K(s)} d_{0},
$$

with $T(s)=\frac{K(s)}{s^{2}+K(s)}=\frac{R(s)}{1+R(s)}$ with $R(s)=K(s) / s^{2}$, taking the form of a complementary sensitivity function. To guarantee $L_{2}$ string stability, with $N$ unboundedly large, it is then necessary in particular that $|T(j \omega)| \leq 1$ at all frequencies $\omega$. This is impossible for a stable system, from the statement of Bode's Complementary Sensitivity integral, which we recall below.

Lemma 1: Assume that the loop transfer function $R(s)$ of a system has (at least) a double pole at $s=0$. If the associated feedback system is stable, then the complementary sensitivity function $T(s)=\frac{R(s)}{1+R(s)}$ must satisfy:

$$
\int_{0}^{\infty} \ln |T(j \omega)| \cdot d \omega / \omega^{2}=\pi \sum_{k} \frac{1}{q_{k}^{(T)}} \geq 0
$$

where $\left\{q_{k}^{(T)}\right\}$ are the zeros of $R(s)$ in the open right half plane. In particular, if $|T(j \omega)|<1$ at some frequencies, then necessarily $|T(j \omega)|>1$ at other frequencies.

This impossibility can be circumvented by using a controller with $h>0$ (and still $W=0$ i.e. no communication, $M^{(r)}=M^{(f)}=1$ i.e. no special sensors), as proposed in Swaroop (1994) and analyzed clearly in Klinge and Middleton (2009). In that case the closed-loop 
dynamics writes

$$
\begin{aligned}
e_{i}= & \frac{K(s)}{s^{2}+(1+h s) K(s)} e_{i-1} \\
& +\frac{1}{s^{2}+(1+h s) K(s)}\left(d_{i-1}-(1+h s) d_{i}\right)
\end{aligned}
$$

and we would have $T(s)=\frac{R(s)}{1+R(s)}$ with $R(s)=\frac{K(s)}{s^{2}+h s K(s)}$, which does not have a double pole at $s=0$ and thus circumvents Lemma 1 . For completeness and later comparison, we give the following result comparable to Klinge and Middleton (2009).

Proposition 1: The norm at $s=j \omega$ of transfer function $T(s)=\frac{K(s)}{s^{2}+(1+h s) K(s)}$ in $(5)$ is $<1$ at all frequencies $\omega \neq 0$, and its $H_{\infty}$ norm equals $T(0)=1$, if and only if one of the following equivalent conditions hold:

(a) If one chooses $\bar{K}(s)=K(s)(1+h s)$ first and then derives $K(s)$ from $h$, then we should ensure that $h$ satisfies

$$
h>\sqrt{\max _{\omega} \frac{\left|\frac{\bar{R}(j \omega)}{1+R(j \omega)}\right|^{2}-1}{\omega^{2}}}
$$

in which $\bar{R}(s)=\bar{K}(s) / s^{2}$.

(b) If one chooses $K(s)$ first, then the criterion becomes

$$
h>\max _{\omega} \sqrt{K_{R}(j \omega)\left(2-\omega^{2} K_{R}(j \omega)\right)}+\omega K_{J}(j \omega)
$$

where $K_{R}(j \omega)=\frac{1}{2}\left(\frac{1}{K(j \omega)}+\frac{1}{K(j \omega)^{*}}\right), K_{J}(j \omega)=\frac{1}{2 j}\left(\frac{1}{K(j \omega)}-\frac{1}{K(j \omega)^{*}}\right)$, and the maximization runs over all $\omega$ for which the argument of the square root is positive.

For particular controllers one can get easy criteria, e.g. for a PD controller $K(s)=b s+a$, it is not hard to see that if $a>2 b^{2}$ the right hand side in case (b) is decreasing with $\omega$, and the condition becomes $h>\sqrt{2 / a}$.

A direct consequence of Prop. 1 is that one can avoid amplifying a disturbance $d_{0}(s)$ along the vehicle chain, and satisfy Definition 2 of string stability. However, remarkably, a corresponding result about Definition 1, i.e. the stronger version of string stability as considered in e.g. Stüdli et al. (2017); Barooah and Hespanha (2005); Seiler et al. (2004), appears to be missing in the literature. The present paper provides the following results in this direction.

Theorem 1: Consider the vehicle chain system (1),(2),(3) with $M^{(r)}=M^{(f)}=1$ and $W=0$. There exists no pair $(K(s), h)$, where $h \geq 0$ is any constant time-headway and $K(s)$ any stabilizing linear controller with $K(0)$ bounded, that would achieve $\left(L_{2}, l_{2}\right)$ norm string stability (Def.1).

Theorem 2: Consider the vehicle chain system (1),(2),(3) with $M^{(r)}=M^{(f)}=1$ and $W=0$. A stabilizing PID controller $K(s)$ with headway $h$ satisfying $(7)$, can ensure $\left(L_{2}, l_{2}\right)$ norm string stability (Def.1).

In light of Thm.1, the lack of positive result about $\left(L_{2}, l_{2}\right)$ string stability in the timeheadway literature can thus be attributed to their focus on bounded stabilizing controllers $K(s)$, excluding e.g. controllers with integral action. Theorem 2 further clarifies that a PID controller indeed does allow to achieve this stronger version of string stability.

The result of Thm.2 obviously covers as well the case where communication is allowed on top of time headway $h>0$. This is the standard setting considered in papers like Öncü et al. (2014); Ploeg et al. (2015), where the communication is meant as a way to improve 
performance rather than just achieving string stability. Those papers also stay with the weaker notion of Def.2, and they impose a more precise communication structure, called Cooperative Adaptive Cruise Control (CACC), by assuming that the message sent by vehicle $i$ to its follower $i+1$ is a filtered version of the input command $u_{i}$ :

$$
q_{i}(s)=\frac{1}{B(s)}\left(K(s) e_{i}(s)+H(s) r_{i}(s)\right),
$$

or in other words (3) with $F=K / B$ and $G=H / B$.

By Thm.2, we have thus established that with $h>0$ and PID control, there exists a communication (namely the trivial one $F=H=G=0$ ) which does achieve the stronger string stability of Def.1 as well. A remaining question is then, how much can communication allow us to weaken some assumptions and still achieve string stability? A negative answer is expressed by the following results.

Theorem 3: Consider the vehicle chain system (1),(2),(3) with $M^{(r)}=M^{(f)}=1$, with the structure (8) of CACC communication.

(a) There exists no $h>0$ and $W, B, H, K$ satisfying Assumption 1 with $K(0)$ bounded, allowing to satisfy $\left(L_{2}, l_{2}\right)$ string stability (Def.1).

(b) For $h=0$, there exists no $W, B, H, K$ satisfying Assumption 1 and allowing to satisfy $L_{2}$ (Def.2) and thus a fortiori $\left(L_{2}, l_{2}\right)$ string stability (Def.1).

Theorem 4: Consider the vehicle chain system (1),(2),(3) with $M^{(r)}=M^{(f)}=1$ and $h=0$. (a) For $K(0)$ bounded, there exist no $W, F, G, H, K$ satisfying Assumption 1, with each $q_{i}$ possibly an $n$-dimensional vector signal for some $n \geq 1$, allowing to satisfy $\left(L_{2}, l_{2}\right)$ string stability (Def.1).

(b) For $q_{i}$ scalar signals, there exist no $W, F, G, H, K$ satisfying Assumption 1 and allowing to satisfy $L_{2}$ (Def.2) and thus a fortiori $\left(L_{2}, l_{2}\right)$ string stability (Def.1).

From Thm.3a, CACC-type communication together with time headway does not exempt us of requiring unbounded $K(0)$, i.e. a result equivalent to Thm.1 still holds. Moreover, Theorems $3 \mathrm{~b}$ and 4 indicate that communication does not allow us to avoid the necessity of time headway; most importantly, in a CACC-type setting or generalized scalar setting, even Def.2 cannot be satisfied. The assumptions a priori still leave a loophole for unbounded $K(0)$ and communicating vector signals $q_{i}$, but we believe that this is just a technical issue and in fact we conjecture that no communication model satisfying Assumption 1 would allow to achieve string stability with (1),(2),(3) and $h=0$.

As a final attempt, we have checked whether we could replace the requirement of time headway $h>0$, by using a relative position measurement that is not just proportional to the true relative position thanks to the dynamics $M^{(r)}, M^{(f)}$. Unfortunately, here too the result is an impossibility.

Theorem 5: Consider the vehicle chain system (1),(2),(3) with $h=0$ and no communication ( $W=G=F=H=0$ ). There exists no choice of stabilizing $K(s)$ and of $M^{(r)}, M^{(f)}$ under the form (4), allowing to satisfy $L_{2}$ string stability (Def.2), and thus a fortiori $\left(L_{2}, l_{2}\right)$ string stability (Def.1).

\section{Conclusion}

We have identified impossibilities to achieve string stability with linear controllers in several extended settings - communication, sensor dynamics, time-headway with bounded DC gain — and one possible solution, namely a PID controller with sufficient time headway. While 
Table 1: known results and clarifications provided by the present paper; with $M^{(r)}=M^{(f)}=1$ unless specified.

\begin{tabular}{|c|c|c|}
\hline $\begin{array}{l}\text { Standard impossibility; Swa- } \\
\text { roop and Hedrick (1996); Swa- } \\
\text { roop (1994); Lemma 1 }\end{array}$ & $h=W=0$ & Def.1 and Def.2 fail \\
\hline $\begin{array}{l}\text { Time-headway solution; Swa- } \\
\text { roop (1994); Klinge and Mid- } \\
\text { dleton (2009); Proposition } 1\end{array}$ & $\begin{array}{l}W=0, h>0 \text { with } \mathrm{PD} \text { controller } \\
(\text { a fortiori also for any } W \neq 0 \text { ) }\end{array}$ & Def.2 holds \\
\hline Theorem 1 & $W=0, h>0$ with $K(0)$ bounded & Def.1 fails \\
\hline Theorem 2 & $\begin{array}{l}W=0, h>0 \text { with PID controller } \\
\text { (a fortiori also for any } W \neq 0 \text { ) }\end{array}$ & $\begin{array}{l}\text { Def.1 and Def.2 } \\
\text { hold }\end{array}$ \\
\hline $\begin{array}{l}\text { CACC with time-headway; } \\
\text { Öncü et al. (2014); Ploeg et al. } \\
(2015)\end{array}$ & $W \neq 0, h>0$ & $\begin{array}{l}\text { Def. } 2 \text { holds with } \\
\text { possibly better scal- } \\
\text { ing, lower } h\end{array}$ \\
\hline Theorem $3 a$ & $\begin{array}{l}\text { CACC-type } W \neq 0, h>0, K(0) \\
\text { bounded }\end{array}$ & Def.1 fails \\
\hline Theorem $3 b$ & CACC-type $W \neq 0, h=0$ & Def.1 and Def.2 fail \\
\hline Theorem $4 a$ & any $W \neq 0, h=0, K(0)$ bounded & Def.1 fails \\
\hline Theorem $4 b$ & any $W \neq 0$, scalar $q_{i}, h=0$ & Def.1 and Def.2 fail \\
\hline Theorem 5 & $W=0, h=0$, tuning $M^{(r)}, M^{(f)}$ & Def.1 and Def.2 fail \\
\hline
\end{tabular}

the proofs do not involve complicated techniques, they do complete the picture about string stability in the strong sense $\left(\left(L_{2}, l_{2}\right)\right.$ bounded reaction to simultaneous perturbations on all the vehicles) and weak sense ( $L_{2}$ bounded reaction of all the vehicles to a disturbance input acting on a single one). We have focused on the standard double-integrator model, i.e. Newton's equation with inputs as only forces, which is not already internally stable; other such models, including MIMO ones, may be worth considering in future work. Regarding extended settings, the paper narrows down the options towards achieving string stability without using absolute velocity. In particular:

- About the use of inter-vehicle communication on a linear channel $W(s)$, we have shown that while this may allow to jointly improve some other performance criteria, it is not a fix on its own for string instability: in all the settings that we have studied, whenever there exists a controller achieving $L_{2}$ or $\left(L_{2}, l_{2}\right)$ string stability with $W \neq 0$, then there also exists a controller achieving it with $W=0$. (The converse of that statement is trivial: if a satisfactory controller is found without using a communication channel, then the presence of a channel $W \neq 0$ cannot hurt since the controller can just choose to ignore the communication messages.)

- Checking typical sensor details, one observes that a distance measurement does not just correspond to a transfer function applied to the actual distance. In other words, actual outputs are not the perfect relative measurements on which the standard string instability proofs rely in an essential way. One might thus wonder whether modeling some sensor details could solve string stability, similarly to and perhaps more gently 
than the use of absolute velocity. We show though that modeling such sensor details does not change the picture for either definition of string stability.

- Regarding bounded DC gain, the bad behavior close to zero frequency is a basic feature and a versatile focus, which should allow to similarly complete the picture for a.o. bidirectional controllers, see Farnam (2018).

These results also highlight the necessity to carefully check in applications whether Def.1, Def.2 or possibly some other string stability notion is the correct proxy for what applications really require. This point has indeed attracted surprisingly little discussion in the literature, although it does appear to change the possible conclusions.

We must end with a short outlook on options that remain open for the controller design itself. One main assumption in this line of literature, including our paper, is of course the use of linear models; nonlinearities and in particular quantization in digital controllers are a priori not covered by the tight impossibility results. However, it appears that this is not a key point. We indeed address this, together with the remaining loophole of vector communication, in ongoing work. Another point regarding communication, is the assumption of local message transmission. If instead all-to-all communication was allowed, e.g. via broadcasting over a communication bus, the picture could be changed. Access to sending over the bus would have to be managed with event-driven decision logic, calling for a comprehensive cyberphysical systems treatment of string stability. One would still have to investigate though, which breakthrough useful information such bus could transmit, when only local distances are measured.

\section{References}

K.C. Chu, "Decentralized control of high-speed vehicular strings," Transportation Science, vol. 8, pp. 361-384, 1974.

S. Stüdli, M.M. Seron and R.H. Middleton, "From vehicular platoons to general networked systems: String stability and related concepts," Annual Reviews in Control, vol. 44, pp. 157$172,2017$.

S. Sheikholeslam and C. Desoer, "Longitudinal control of a platoon of vehicles," Proc. American Control Conf., pp. 291-297, 1990.

W. Levine and M. Athans, "On the optimal error regulation of a string of moving vehicles," IEEE Trans. Automatic Control, vol. 11, pp. 355-361, 1996.

J.A. Rogge and D. Aeyels, "Vehicle platoons through ring coupling," IEEE Trans. Automatic Control, vol. 53, pp. 1370-1377, 2008.

D. Swaroop and J. Hedrick, "String stability of interconnected systems," IEEE Trans. Automatic Control, vol. 41, pp. 349-357, 1996.

J. Ploeg, D.P. Shukla, N. van de Wouw and H. Nijmeijer, "Controller synthesis for string stability of vehicle platoons," IEEE Trans.Intell.Transp. Systems, vol. 15, pp. 854-865, 2014. 
D. Swaroop, "String stability of interconnected systems: An application to platooning in automated highway systems," PhD thesis, University of California, Berkeley, 1994.

P. Barooah and J. P. Hespanha, "Error amplification and disturbance propagation in vehicle strings with decentralized linear control," Proc. IEEE Conf. on Decision and Control, pp. 4964-4969, 2005.

P. Barooah, P. G. Mehta and J. P. Hespanha, "Mistuning-based control design to improve closed-loop stability of vehicular platoons," IEEE Trans. Automatic Control, vol. 54, pp. 2100-2113, 2009.

P. Seiler, A. Pant, and K. Hedrick, "Disturbance propagation in vehicle strings," IEEE Trans. Automatic Control, vol. 37, pp. 1835-1842, 2004.

H. Hao and P. Barooah, "Stability and robustness of large platoons of vehicles with doubleintegrator models and nearest neighbor interaction," Int.J. Robust and Nonlinear Control, vol. 23, pp. 2097-2122, 2013.

Y. Yamamoto, and M.C. Smith, "Bounded disturbance amplification for mass chains with passive interconnection," IEEE Trans. Automatic Control, vol. 47, pp. 2534-2542, 2015.

A. Farnam, "Towards impossibility and possibility results for string stability of platoon of vehicles", PhD thesis, Ghent University, Belgium, 2018.

S. Klinge, and R.H. Middleton, "Time headway requirements for string stability of homogenous linear unidirectionally connected systems," Proc. IEEE Conf. on Decision and Control, pp. 1992-1997, 2009.

S. Knorn, A. Donaire, J.C. Aguero b and R.H. Middleton, "Passivity-based control for multivehicle systems subject to string constraints," Automatica, vol. 50, pp. 3224-3230, 2014.

S. Öncü, J. Ploeg, N. van de Wouw, and H. Nijmeijer, "Cooperative Adaptive Cruise Control: network-aware analysis of string stability," IEEE Trans.Intell.Transp. Systems, vol. 15, pp. 1527-1537, 2014.

J. Ploeg, N. van de Wouw, and H. Nijmeijer, " $L_{p}$ string stability of cascaded systems: application to vehicle platooning," IEEE Trans. Control Systems Technology, vol. 22, pp. 786-793, 2014 .

J. Ploeg, E. S. Kazerooni, G. Lijster, N. van de Wouw, and H. Nijmeijer, "Graceful degradation of Cooperative Adaptive Cruise Control," IEEE Trans.Intell.Transp. Systems, vol. 16, pp. 488-796, 2015.

V. Milanés and S. E. Shladover, "Modeling cooperative and autonomous adaptive cruise control dynamic responses using experimental data," Transportation Research Part C: Emerging Technologies, vol. 48, pp. 285-300, 2014.

I. Serban and M. Najim, "Multidimensional systems: BIBO stability test based on functional Schur coefficients," IEEE Trans. Signal Processing, vol. 55, pp. 5277-5285, 2007.

Y. Li and B. De. Schutter, "Control of a string of identical pools using non-identical feedback controllers," IEEE Trans. Control Systems Technology, vol. 20, pp. 1638-1646, 2012. 
I. Herman, S. Knorn and A. Ahlén, "Disturbance scaling in bidirectional vehicle platoons with different asymmetry in position and velocity coupling," Automatica, vol. 82, pp. 13$20,2017$.

\section{A Proofs}

Proof of Proposition 1: For case (a), we reformulate $T(s)=\frac{1}{1+h s} \frac{\bar{K}(s) / s^{2}}{1+\bar{K}(s) / s^{2}}$. Then writing

$$
|T(j \omega)|^{2}=\frac{1}{1+\omega^{2} h^{2}}\left|\frac{R(j \omega)}{1+R(j \omega)}\right|^{2}<1 \text { for all } \omega \neq 0
$$

directly yields the expression, where the Bode integral (Lemma 1) ensures that the max inside the square root will be non-negative. For case (b), we just write $1 /|T(j \omega)|^{2}=\mid-\omega^{2} / K(j \omega)+$ $\left.(1+h j \omega)\right|^{2}>1$ and we group real and imaginary parts to isolate $h$.

Proof of Theorem 1: We take in particular a disturbance input $d_{0}$ that affects the leading vehicle only. From (5), such disturbance leads to

$$
e_{i}=T(s)^{i-1} \frac{1}{s^{2}+(1+h s) K(s)} d_{0} \quad, \quad i=1,2, \ldots, N,
$$

with $T(s)=\frac{K(s)}{s^{2}+(1+h s) K(s)}$ as in Proposition 1. Then

$$
\sum_{i=1}^{N}\left|e_{i}(s)\right|^{2}=\sum_{i=0}^{N-1}|T(s)|^{2 i} \cdot \frac{\left|d_{0}(s)\right|_{2}^{2}}{\left|s^{2}+(1+h s) K(s)\right|^{2}} .
$$

Consider a disturbance concentrated at low frequencies, such that $\int_{-\epsilon}^{\epsilon}\left|d_{0}(j \omega)\right|^{2} d \omega \geq \frac{1}{2} \int_{-\infty}^{+\infty}\left|d_{0}(j \omega)\right|^{2} d \omega$ for some $\epsilon \ll 1$. Writing

$$
\|e(.)\|_{2}^{2} \geq \int_{-\epsilon}^{\epsilon} \sum_{i=1}^{N}\left|e_{i}(j \omega)\right|^{2} d \omega,
$$

we lower-bound the right hand side thanks to continuity at $\omega=0$. Indeed, in (9), for $K(0)$ finite, there exist $\delta, \alpha>0$ such that $\left.\frac{1}{\left|s^{2}+(1+h s) K(s)\right|^{2}}\right|_{s=j \omega}>\alpha$ for all $\omega \in(-\delta, \delta)$. Moreover, since $T(0)=1$, we can also make $\min _{\omega \in[0, \epsilon)}|T(j \omega)|$ arbitrarily close to 1 by taking $\epsilon$ close enough to 0 . Therefore, we can make the right hand side of (10) arbitrarily close to $\frac{N \alpha}{2}\left\|d_{0}(.)\right\|_{2}^{2}$ by concentrating $d_{0}$ on low enough frequencies $\epsilon<\delta$. Thus the factor relating $\|e(.)\|_{2}^{2}$ to $\left\|d_{0}(.)\right\|_{2}^{2}=\|d(.)\|_{2}^{2}$ cannot be bounded independently of the disturbance signal $d($. and of $N$.

Proof of Theorem 2: For this positive result we must prove that we can tune the gains such that the system is stable, and simultaneously we can guarantee $\|e(.)\|_{2}<C_{0}\|d(.)\|_{2}$ in which the constant $C_{0}$ is bounded independently of number of vehicles $N$. We split the proof in two parts. First, we show that there exists a set of PID controller tuning and of values of $h$, for which the system is stable while satisfying Proposition 1 (i.e. the $L_{2}$ stability criterion). Second, we show that this also allows to satisfy $\left(L_{2}, l_{2}\right)$ string stability.

For stability under the conditions of Proposition 1: Considering the first criterion in Proposition 1, we would fix some tuning of the polynomial $\bar{K}(s)$ which makes the system 
stable (just checking always the same denominator $s^{3}+s(1+h s) K(s)=s^{3}+s \bar{K}(s)$ ). Once $\bar{K}(s)$ has been selected, we would then choose $h$ according to the related criterion, while adapting the other parameters in order to maintain $\bar{K}(s)$ fixed as selected. For this to be possible, the only essential element is to prove that $h$ in the first criterion of Proposition 1 always remains bounded for a stable PID controller.

We thus consider $s^{3}+s \bar{K}(s)$ to be any third-order polynomial with roots in the open left half plane. Then in the criterion,

$$
\frac{\bar{R}}{1+\bar{R}}=\frac{s \bar{K}(s)}{s^{3}+s \bar{K}(s)}
$$

remains bounded for all $s=j \omega$ and we must only investigate the behavior for $\omega$ close to 0 . From the inverse triangle inequality $\left|\frac{\bar{R}}{1+\bar{R}}\right|^{2}-1 \leq\left|\left(\frac{\bar{R}}{1+\bar{R}}\right)^{2}-1\right|$ a sufficient criterion for Proposition 1(a) is

$$
h>\sqrt{\left|\frac{(j \omega)^{2} \bar{K}^{2}(j \omega)-\left[(j \omega)^{3}+(j \omega) \bar{K}(j \omega)\right]^{2}}{\omega^{2}\left[(j \omega)^{3}+(j \omega) \bar{K}(j \omega)\right]^{2}}\right|},
$$

which just comes down to

$$
h>\sqrt{\left|\frac{\omega^{4}-2 \omega^{2} K(j \omega)}{\left(j \omega \bar{K}(j \omega)-j \omega^{3}\right)^{2}}\right|} .
$$

For $\omega$ close to 0 and $K(j \omega)$ a PID controller, the dominating term is $h>\sqrt{\left|2 \omega k_{I} / k_{I}^{2}\right|}$, with $k_{I}$ the integral gain. This imposes a bounded constraint on $h$ and it is thus possible indeed to satisfy stability and the criterion of Proposition 1 simultaneously with a PID controller.

For $\left(L_{2}, l_{2}\right)$ string stability, we write in matrix form:

$$
e(s)=(-L(s) \mathbf{A}+L(s) \mathbf{B}(s)+P(s) \mathbf{C}(s)) d(s)
$$

with $P(s)=\frac{s^{2}+h s K(s)}{\left(s^{2}+(1+h s) K(s)\right)^{2}}, L(s)=\frac{1}{s^{2}+(1+h s) K(s)}$ and the $N \times(N+1)$ matrices

$$
\begin{aligned}
\mathbf{A}= & {\left[\begin{array}{ccccc}
0 & 1 & 0 & \ldots & 0 \\
0 & 0 & 1 & \ldots & 0 \\
0 & 0 & 0 & \ldots & 0 \\
\vdots & \vdots & \vdots & \vdots & \vdots \\
0 & 0 & 0 & \ldots & 1
\end{array}\right], \mathbf{B}(s)=\left[\begin{array}{ccccc}
1 & 0 & 0 & \ldots & 0 \\
T(s) & 0 & 0 & \ldots & 0 \\
T(s)^{2} & 0 & 0 & \ldots & 0 \\
\vdots & \vdots & \vdots & \vdots & \vdots \\
T(s)^{N-1} & 0 & 0 & \ldots & 0
\end{array}\right] } \\
\mathbf{C}(s)= & {\left[\begin{array}{ccccc}
0 & 0 & 0 & \ldots & 0 \\
0 & 1 & 0 & \ldots & 0 \\
0 & T(s) & 1 & \ldots & 0 \\
\vdots & \vdots & \vdots & \vdots & \vdots \\
0 & T(s)^{N-2} & T(s)^{N-3} & \ldots & 0
\end{array}\right] . }
\end{aligned}
$$

By the triangle inequality,

$$
\begin{aligned}
\|e(s)\|_{2} \leq & \left(|L(s)|\|\mathbf{A}\|_{2}+|L(s)|\|\mathbf{B}(s)\|_{2}\right. \\
& \left.+|P(s)|\|\mathbf{C}(s)\|_{2}\right)\|d(s)\|_{2}
\end{aligned}
$$


with the induced matrix norms $\|\mathbf{D}\|_{2}=\sqrt{\lambda_{\max }\left(\mathbf{D}^{*} \mathbf{D}\right)}$ where ${ }^{*}$ is the complex conjugate transpose. The proof now comes down to proving a bounded norm, independent of $N$ and $s=j \omega$, for each of the three terms in front of $\|d(s)\|_{2}$ in (11).

For the first term, since $\mathbf{A}^{*} \mathbf{A}=\operatorname{diag}(0,1,1,1, \ldots, 1)$, we immediately have $|L(s)|\|\mathbf{A}\|_{2}=$ $|L(s)|$, and the latter can be bounded independently of $s=j \omega$ for a stable system.

For the second term, we have $\mathbf{B}^{*} \mathbf{B}=\operatorname{diag}\left(\frac{1-|T(s)|^{2 N}}{1-\left|T(s)^{2}\right|}, 0,0, \ldots, 0\right)$. Under the conditions of Prop.1, the numerator is lower than 1 and $|L(j \omega)|\|\mathbf{B}(j \omega)\|_{2} \leq \sqrt{\frac{|L(j \omega)|^{2}}{1-|T(j \omega)|^{2}}}$. The unbounded DC gain ensures that, when $T(j \omega)-1$ converges to 0 at $\omega=0$, so does $L(j \omega)$. Analysis close to $\omega=0$ yields

$$
\frac{|L(j \omega)|^{2}}{1-|T(j \omega)|^{2}} \simeq \frac{\omega^{2}}{k_{I}^{2}} \cdot \frac{1}{h^{2} \omega^{2}}=\frac{1}{k_{I}^{2} h^{2}}
$$

i.e. the limit for $\omega \rightarrow 0$ of $|L(j \omega)|\|\mathbf{B}(j \omega)\|_{2}$ is bounded, independently of $N$. It is then easy to find a bound that is valid at all frequencies $\omega$, independently of $N$.

To bound the third term, we can use the Gershgorin disk theorem on the matrix $|P|^{2} \mathbf{C}^{*} \mathbf{C}$. For $|T(j \omega)| \leq 1$ we can bound finite sums of powers of $|T(j \omega)|$ by an infinite geometric series, and trivially check that the eigenvalues are bounded independently of $N$, for all $\omega$ outside a neighborhood of the origin $\omega=0$. The latter is indeed the only place where $|T(j \omega)|=1$, and an expansion for $\omega \ll 1$ shows that in fact $|P(j \omega)|^{2}\|\mathbf{C}(j \omega)\|_{2}^{2}$ converges to zero for $\omega \rightarrow 0$. This concludes the proof.

Proof of Theorem 3: Defining $z_{i}=\left[e_{i} ; q_{i-1}-q_{i}\right]$, the closed-loop dynamics with $d_{0} \neq 0$ only is described by:

$$
\begin{aligned}
& z_{i+1}=\mathbf{T}(s) z_{i}, \quad z_{1}=\left[\begin{array}{l}
\frac{1}{s^{2}+K(1+h s)} \\
\frac{-K / B}{s^{2}+K(1+h s)}
\end{array}\right] d_{0} \\
& \text { with } \mathbf{T}(s)=\left[\begin{array}{ll}
\frac{K}{s^{2}+K(1+h s)} & \frac{H W}{s^{2}+K(1+h s)} \\
\frac{K}{B} \cdot \frac{s^{2}}{s^{2}+K(1+h s)} & \frac{H W}{B} \cdot \frac{s^{2}}{s^{2}+K(1+h s)}
\end{array}\right] .
\end{aligned}
$$

The key simplification implied by CACC is that $\mathbf{T}(s)$ is singular for all $s$, since the right column equals $H W / K$ times the left column. Thus the single nonzero eigenvalue of $T(s)$ equals its trace, $\operatorname{trace}(\mathbf{T}(s))=\frac{K+\frac{H W}{B} s^{2}}{K(1+h s)+s^{2}}$.

(a) For any $h>0$, trace $(\mathbf{T}(s))$ approaches 1 when $s$ approaches 0 . Thus like in the proof of Thm.1, a uniform bound over $\omega, N$ cannot be found for $\|e\|_{2}$, if the corresponding mode with $s=0$ has a nonzero component in $z_{1}$. The corresponding eigenvector at $s=0$ is $z_{i} \propto[1 ; 0]$, while the zero eigenvector is $z_{i} \propto[1 ;-K(0) / H W(0)]$. Thus $z_{1}$ will have a nonzero component on the "bad" mode unless $z_{1} \propto[1 ;-K / B(0)] \propto[1 ;-K(0) / H W(0)]$, i.e. either $K(0)$ unbounded which we exclude by assumption, or $H W(0)=B(0)$ exactly. The latter is forbidden by the last requirement of Assumption 1.

(b) For $h=0$, we can rewrite $\operatorname{trace}(\mathbf{T}(s))=\frac{R}{1+R}$ with $R=\frac{K+\frac{H W}{B} s^{2}}{s^{2}(1-H W / B)}$. Since $\frac{H W}{B}$ is bounded, the denominator decays at least as $s^{2}$ for $s$ close to 0 and we are in the conditions to apply Lemma 1 (Bode complementary sensitivity integral); this implies that there will be a range of frequencies $\omega$ where $\mathbf{T}(j \omega)$ has an eigenvalue with norm $|\operatorname{trace}(\mathbf{T}(j \omega))|$ larger than 1 . As for case (a), with the last requirement of Assumption 1 the system will unavoidably have a component of $z_{1}(\omega)$ on this mode, which unavoidably makes the system string unstable in the sense of Def.2. 
Proof of Theorem 4: Similarly to the case of CACC, and somewhat simplified thanks to $h=0$, by defining $z_{i}=\left[e_{i} ; q_{i-1}\right]$ we can reformulate the dynamics as:

$$
\begin{aligned}
& z_{i+1}=\mathbf{T}(s) z_{i}+\left[\begin{array}{l}
\frac{1}{s^{2}+K} \\
0
\end{array}\right]\left(d_{i}-d_{i+1}\right) \\
& \text { with } \mathbf{T}(s)=\left[\begin{array}{ll}
\frac{K-H W F}{s^{2}+K} & \frac{H W(I-G W)}{s^{2}+K} \\
F & G W
\end{array}\right] .
\end{aligned}
$$

Here $I$ is the identity matrix, emphasizing that $q_{i}$ might be a vector and $F, G, H, W$ appropriate matrices.

For case (a) the proof follows the same lines as Thm.3(a), after checking that $\mathbf{T}(\mathbf{0})$ has an eigenvalue 1.

For case (b) we use a Routh-Hurwitz type criterion for discrete systems, see e.g. Serban and Najim (2007). For a two-dimensional state matrix $A$, it states that the eigenvalues belong to the unit circle provided

- $|\operatorname{det}(A)| \leq 1$ and

- $\operatorname{det}(A)^{*} \operatorname{trace}(A)-\left.\operatorname{trace}(A)^{*}|\leq 1-| \operatorname{det}(A)\right|^{2}$.

The determinant of $\mathbf{T}(s)$ imposes

$$
|\operatorname{det}|=\left|\frac{G W K-H W F}{s^{2}+K}\right|=: \frac{|A|}{\left|s^{2}+K\right|} \leq 1,
$$

where we have defined $A=(G K-H F) W$. Next, we need

$$
\begin{aligned}
1 & \geq \frac{\mid \text { trace }- \text { trace*det } \mid}{1-|\operatorname{det}|^{2}} \\
& =\left|1+\frac{s^{2}}{1-|\operatorname{det}|^{2}}\left(\frac{G W-1}{s^{2}+K}-\frac{(G W-1)^{*}}{\left(s^{2}+K\right)^{*}} \frac{A}{s^{2}+K}\right)\right| .
\end{aligned}
$$

Since $\frac{s^{2}}{1-|\operatorname{det}|^{2}}$ is real negative for $s=j \omega$ and $|\operatorname{det}|<1$, the above equation cannot be satisfied if $(G W-1) /\left(s^{2}+K\right)$ takes a real negative value for some $s=j \omega$. Indeed, for any $c_{1}, c_{2}$ real negative and $c_{3}$ complex but of norm smaller than one, we have that $1+c_{1} c_{2}\left(1-c_{3}\right)$ lies outside the unit disk. Thus to conclude the proof, there remains to show that $(G W-1) /\left(s^{2}+K\right)$ will always take a real negative value for some $s=j \omega$.

Since $s^{2}+K$ has two more zeros than poles, and all zeros must satisfy stability, we have that the phase Bode plot of $1 /\left(s^{2}+K\right)$ goes down at least by 180 degrees, to end at -180 degrees for $\omega$ tending to infinity. In contrast, $G W-1$ has as many zeros as poles; all poles are stable, implying 90 degrees down in the phase Bode plot, such that overall with $G W-1$ we either go down or stay, and again we end at -180 degrees for $\omega$ tending to infinity. Now assume as a first possibility, that $G W-1$ starts at another value than -180 degrees. In this case, it must go down nontrivially, i.e. we must go sown by strictly more than 180 degrees to end up at -360 degrees: somewhere in between, there will be a 180 degree phase, proving impossibility. (Note indeed that we forbid any perfect cancellation with $G W=1$ at a target value of $\omega$.) So the only choice left is that $G W$ starts at -180 degrees. Then for $K(0)$ finite we would have a negative real phase at $s=0$, thus impossible. There remains the case with $K$ having a pole of order $m>0$ at $s=0$. In this case, $1 /\left(s^{2}+K\right)$ has $m$ of its zeros at $s=0$, and $\frac{1}{s^{m}} \frac{1}{s^{2}+K}$ has a phase Bode plot going down by $(180+m 90)$ degrees overall. This means, 
$\frac{G W-1}{s^{2}+K}$ would start with a phase of $-180+m 90$ degrees at $s=0$, then go down by $180+m 90$ degrees to end up at -360 degrees for $\omega$ tending to infinity, with $m>0$. Again, this implies a phase of -180 degrees for some intermediate $\omega$. There are no possibilities left, so the proof is concluded.

Proof of Theorem 5: The error dynamics write

$$
e_{i}=M^{(r)} \cdot \frac{1}{M^{(f)}} \cdot T^{\prime}(s) e_{i-1}=: A(s) e_{i-1}
$$

where $T^{\prime}(s)=\frac{K^{\prime}(s)}{s^{2}+K^{\prime}(s)}$ with $K^{\prime}(s)=M^{(f)}(s) \cdot K(s)$. With $(4), M^{(r)}, M^{(f)}$ and $T^{\prime}$ all take the form of complementary sensitivity functions satisfying Lemma 1 . We thus have

$$
\int_{0}^{\infty} \ln |A(j \omega)| \cdot d \omega / \omega^{2}=\sum_{k} \frac{1}{q_{k}^{\left(M^{(r)}\right)}}-\frac{1}{q_{k}^{\left(M^{(f)}\right)}}+\frac{1}{q_{k}^{\left(T^{\prime}\right)}},
$$

with the $q$ 's denoting the respective zeros of the loop transfer functions in the right half plane. Having a control effect requires $|A(j \omega)|<1$ at some frequencies, while having $|A(j \omega)|>1$ at any frequency would imply that the system is string unstable. Combining these two features requires that the right hand side be negative. The only way to obtain this is if $K^{(f)}(s) / s^{2}$ has zeros in the open right half plane, without having the same zeros in the other terms. However, the latter would mean that $M^{(f)}(s)$ has zeros in the right half plane, unmatched by the other transfer functions, and by (12) this would imply that the vehicle chain has a pole in the right half plane i.e. it is unstable. 